\title{
Hepatocyte Growth Factor Activator Inhibitor Type 1 Maintains the Assembly of Keratin into Desmosomes in Keratinocytes by Regulating Protease- Activated Receptor 2-Dependent p38 Signaling
}

Makiko Kawaguchi, ${ }^{*}$ Ai Kanemaru, ${ }^{*}$ Akira Sawaguchi, ${ }^{\dagger}$ Koji Yamamoto, ${ }^{\ddagger}{ }^{\ddagger}$ Takashi Baba,${ }^{\ddagger}$ Chen-Yong Lin, ${ }^{\S}$ Michael D. Johnson, Tsuyoshi Fukushima, ${ }^{*}$ and Hiroaki Kataoka*

From the Section of Oncopathology and Regenerative Biology, ${ }^{*}$ Department of Pathology, the Section of Ultrastructural Cell Biology, ${ }^{\dagger}$ Department of Anatomy, and the Department of Oral and Maxillofacial Surgery, ${ }^{\ddagger}$ Faculty of Medicine, University of Miyazaki, Miyazaki, Japan; and the Department of Oncology, ${ }^{\S}$ Lambardi Comprehensive Cancer Centre, Georgetown University, School of Medicine, Washington, District of Columbia

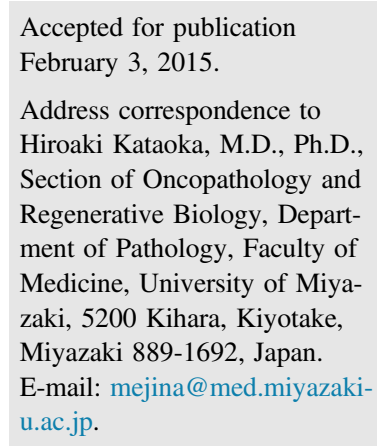

Address correspondence to Hiroaki Kataoka, M.D., Ph.D., Section of Oncopathology and Regenerative Biology, Department of Pathology, Faculty of Medicine, University of Miyazaki, 5200 Kihara, Kiyotake, Miyazaki 889-1692, Japan. E-mail: mejina@med.miyazakiu.ac.jp.

\begin{abstract}
Hepatocyte growth factor activator inhibitor type 1 (HAI-1; official symbol SPINT1) is a membraneassociated serine proteinase inhibitor abundantly expressed in epithelial tissues. Genetically engineered mouse models demonstrated that HAI-1 is critical for epidermal function, possibly through direct and indirect regulation of cell surface proteases, such as matriptase and prostasin. To obtain a better understanding of the role of HAI-1 in maintaining epidermal integrity, we performed ultrastructural analysis of Spint1-deleted mouse epidermis and organotypic culture of an HAI-1 knockdown (KD) human keratinocyte cell line, HaCaT. We found that the aggregation of tonofilaments to desmosomes was significantly reduced in HAI-1-deficient mouse epidermis with decreased desmosome number. Similar findings were observed in HAI-1 KD HaCaT organotypic cultures. Immunoblot and immunohistochemical analyses revealed that p38 mitogen-activated protein kinase was activated in response to HAI-1 insufficiency. Treatment of HAI-1 KD HaCaT cells with a p38 inhibitor abrogated the above-observed ultrastructural abnormalities. The activation of p38 induced by the loss of HAI-1 likely resulted from enhanced signaling of protease-activated receptor-2 (PAR-2), because its silencing abrogated the enhanced activation of p38. Consequently, treatment of HAI-1 KD HaCaT cells with a serine protease inhibitor, aprotinin, or PAR-2 antagonist alleviated the abnormal ultrastructural phenotype in organotypic culture. These results suggest that HAI-1 may have a critical role in maintaining normal keratinocyte morphology through regulation of PAR-2-dependent p38 mitogen-activated protein kinase signaling. (Am J Pathol 2015, 185: 1610-1623; http://dx.doi.org/10.1016/j.ajpath.2015.02.009)
\end{abstract}

Human skin protects the body from both water loss and mechanical damage. This barrier function is primarily accomplished by the epidermis, a self-renewing stratified squamous epithelium composed of several layers of keratinocytes. ${ }^{1}$ To achieve the barrier functions, keratinocytes form dynamic and strong cell-cell junctions in which desmosomes and the network of keratin intermediate filaments (KIFs) are essential to maintain junctional integrity. ${ }^{2}$ KIFs are anchored to the cytoplasm and interact with desmosomal cell-cell contacts at the plasma membrane. These are important for mechanical stability of cell-cell contacts between keratinocytes. ${ }^{3}$ Disturbance of the integrity of the KIF network leads to skin-blistering diseases, such as epidermolysis bullosa simplex. ${ }^{4}$ Although it is not known how KIF disassembly is regulated, recent studies suggested that p38 mitogen-activated protein kinase (MAPK) signaling is involved in these processes. ${ }^{4-6}$

The p38 is a member of the MAPK family. It is present in epithelial cells, responds rapidly to various types of stresses,

\footnotetext{
Supported by Ministry of Education, Science, Sports and Culture, Japan, grants-in-aid for scientific research 24390099 (H.K.) and 25860166 (M.K.) Disclosures: None declared.
} 
and is activated by proinflammatory cytokines. ${ }^{7,8}$ It is known that $\mathrm{p} 38$ MAPK is increased in psoriatic skin, and it is rapidly phosphorylated in response to pemphigus vulgaris $\mathrm{IgG}^{5}$ Activated p38 promotes KIF retraction and disruption of desmosome-mediated cell-cell adhesion. ${ }^{6}$ Desmosomes consist of two desmosome-specific cadherin family members, desmogleins and desmocollins, as well as a collection of cytoplasmic plaque proteins, including desmoplakin, plakoglobin, and plakophilins. ${ }^{9}$ Previous studies showed that these proteins are degraded by serine proteases. For example, matriptase, a type II transmembrane serine protease on the epithelial cell surface, degrades desmoglein-2, and kallikrein 1-related peptidase (KLK)-5 promotes cleavage of desmoglein-1. ${ }^{10,11}$

Hepatocyte growth factor activator inhibitor type 1 (HAI-1; official symbol SPINT1), encoded by the SPINT1 gene, is a serine protease inhibitor abundantly expressed in the placenta and in epithelial tissues. ${ }^{12,13}$ HAI-1 regulates several trypsinlike serine proteinases, such as hepatocyte growth factor activator, matriptase, prostasin, hepsin, TMPRSS13, human airway trypsin-like protease, and KLKs 4 and $5 .{ }^{14-20}$ By using Spint 1 mutant mouse models, we previously reported that HAI-1 is critically required in the development of the placental labyrinth $^{21}$ and normal keratinization of the skin, ${ }^{22}$ and it may also contribute to intestinal epithelial barrier function. ${ }^{23}$ In the absence of HAI-1, epidermis showed hyperkeratosis and decreased barrier function in mice. ${ }^{22}$ Moreover, hair cuticle formation was severely impaired. ${ }^{22}$ More important, these skin pathologies caused by HAI-1 deficiency were totally abrogated in the matriptase hypomorphic mice, ${ }^{24}$ indicating that HAI-1 is a critical regulator of matriptase in the skin. Matriptase is also known to activate other serine proteases, such as prostasin and KLK-5. ${ }^{25,26}$ Insufficient HAI-1 function on the cell surface would result in a severely deranged pericellular proteolysis network that could significantly influence cellular function.

Protease-activated receptor 2 (PAR-2) is a G proteincoupled receptor that is able to mediate multiple intracellular signaling pathways on cleavage of its activation site by a trypsin-like serine protease. ${ }^{27}$ In the skin, PAR-2 is widely expressed by almost all cell types, especially keratinocytes. It has been implicated in the regulation of keratinocyte proliferation and differentiation, epidermal barrier function, and inflammation. ${ }^{27-29}$ Recent studies have revealed that matriptase and prostasin are important activators of PAR-2 in the skin. For example, matriptase-driven premalignant progression is prevented by genetic elimination of PAR-2, and a prostasin-induced ichthyosis-like skin phenotype is rescued by concomitant deletion of PAR-2. ${ }^{30,31}$ Therefore, it is reasonable to speculate that HAI-1 regulates PAR-2 function through regulation of PAR-2-activating serine proteases in keratinocytes, a relationship that may have significant impact on epidermal integrity.

This study aimed to address the role of HAI-1 in the regulation of epidermal integrity. We used Spint1-deleted mouse skin tissues, ${ }^{22}$ primary culture of Spint 1 -deleted mouse keratinocytes, and an HAI-1 knockdown (KD) human keratinocyte cell line, $\mathrm{HaCaT}$. We found that insufficient HAI-1 in keratinocytes resulted in abnormal KIF assembly. This abnormality was caused by activation of p38 MAPK, which was induced by enhanced PAR-2 activation by protease(s) regulated by HAI- 1 . These results suggest that HAI-1 has an important role in maintaining normal keratinocyte morphology and cell-cell adhesion through suppression of PAR-2 activation.

\section{Materials and Methods}

\section{Antibodies and Reagents}

The following antibodies were used: anti-matriptase mouse monoclonal antibody (mAb; M24), ${ }^{32}$ anti-activated matriptase mouse mAb (M69), ${ }^{32}$ anti-HAI-1 goat polyclonal antibody (R\&D Systems, Minneapolis, MN), anti-prostasin mouse mAb (BD Bioscience, San Jose, CA), anti-PAR-2 mouse mAb SAM11 (Santa Cruz Biotechnology, Santa Cruz, CA), anti-p38 and anti-phosphorylated p38 MAPK (Thr180/Tyr182) rabbit mAbs (Cell Signaling Technology Japan, Tokyo, Japan), Alexa Fluor 594-conjugated anti-phosphorylated p38 MAPK (Thr180/Tyr182) rabbit mAbs (Cell Signaling Technology), anti-AKT and anti-phosphorylated AKT (Ser473) rabbit mAbs (Cell Signaling Technology), anti-p44/42 MAPK [extracellular signal-regulated kinase (ERK) 1/2] rabbit polyclonal antibody and anti-phosphorylated ERK1/2 (Thr202/Tyr204) rabbit mAb (Cell Signaling Technology), anti-cytokeratin (CK) 5 rabbit mAb (Abcam, Cambridge, UK), anti-CK10 mouse mAb (Dako, Tokyo, Japan), and anti-desmoglein 3 mouse $\mathrm{mAb}$ (Life Technologies Japan, Tokyo, Japan). Aprotinin and specific p38 inhibitor, SB203580, were purchased from Sigma (St. Louis, MO). A broad-spectrum metalloprotease inhibitor, GM6001, was from Millipore (Billerica, MA). Recombinant human matriptase catalytic domain was obtained from R\&D Systems. PAR-2-activating peptide, SerLeu-Ile-Gly-Arg (SLIGR)-NH $\mathrm{N}_{2}$, and PAR-2 selective antagonist, Phe-Ser-Leu-Leu-Arg-Tyr (FSLLRY)-NH $\mathrm{N}_{2}$, were purchased from Peptides International (Louisville, KY).

\section{Spint1-Deleted Mouse Skin}

Spint $^{-/-}$mice were generated by injection of Spint $1^{+/+}$ blastocyst with Spint1 ${ }^{-1-}$ embryonic stem cells (C57BL/6 background), as described previously. ${ }^{22}$ C57BL/6 wild-type mice were used as a control. After mice were sacrificed with diethyl ether, full-thickness dorsal skin samples were immediately removed and processed for primary culture of keratinocytes, immunohistological analysis, or ultrastructural analysis. All animal experiments were approved by the Animal Care and Use Committee of the University of Miyazaki (Miyazaki, Japan).

\section{Cell Cultures}

Primary murine keratinocytes were prepared as described previously. ${ }^{22}$ Cells were cultured in an epidermal keratinocyte medium (CELLnTEC Advanced Cell Systems, Bern, 
Table 1 Primer Sequences Used for Real-Time RT-PCR

\begin{tabular}{|c|c|c|c|}
\hline Target & Forward primer & Reverse primer & Size $(b p)$ \\
\hline$\beta$-Actin & $5^{\prime}-$ ATTGCCGACAGGATGCAGA-3' & 5'-GAGTACTTGCGCTCAGGAGGA-3' & 89 \\
\hline HAI-1 & 5'-CAGCAGTGCCTCGAGTCTTGTC-3' & 5'-GATGGCTACCACCACCACAATG-3' & 144 \\
\hline Matriptase & $5^{\prime}$-CTTTGAGGCCACCTTCTTC-3' & 5'-GGTAGTGGCCTGGGTAGTA-3' & 104 \\
\hline Desmoglein 3 & $5^{\prime}$-CTTCAGATTACCAAGCAACCCAGA-3' & 5'-GGGCATTTAGAGCCCGACA-3' & 177 \\
\hline
\end{tabular}

HAI-1, hepatocyte growth factor activator inhibitor type 1.

Switzerland). HaCaT cells were purchased from the CLS Cell Line Service GmbH (Eppelheim, Germany) and maintained in Dulbecco's modified Eagle's medium (DMEM) supplemented with $10 \%$ fetal bovine serum (FBS), penicillin, and streptomycin. A human dermal fibroblast cell line, SF-TY, was obtained from the Japanese Collection of Research Bioresources Cell Bank (Osaka, Japan). In our three-dimensional (3D) organotypic skin model, $2.5 \times 10^{4}$ SF-TY cells suspended in $0.1 \mathrm{~mL}$ of FBS were mixed with 1 $\mathrm{mL}$ of DMEM containing $2.2 \mathrm{mg} / \mathrm{mL}$ type I collagen
(KOKEN Co, Tokyo, Japan), $10 \mathrm{mmol} / \mathrm{L}$ HEPES, and 10 $\mathrm{mmol} / \mathrm{L} \mathrm{NaHCO} 3$, which was transferred into a 12-well cellculture insert (pore size, $0.4 \mu \mathrm{m}$; Millipore). After incubation in DMEM with $10 \%$ FBS overnight, $5 \times 10^{5} \mathrm{HaCaT}$ cells were placed on the fibroblast-containing collagen gel and incubated for 24 hours. Then, the gel surface was raised to the air-liquid interface and cultured for 3 weeks to allow $\mathrm{HaCaT}$ cells to stratify and differentiate. The culture medium ( $1 \mathrm{~mL}$ per well) was changed every 3 days, and in an indicated experiment, $1 \mu \mathrm{L}$ of $\mathrm{SB} 203580(10 \mathrm{mmol} / \mathrm{L}$ in
A
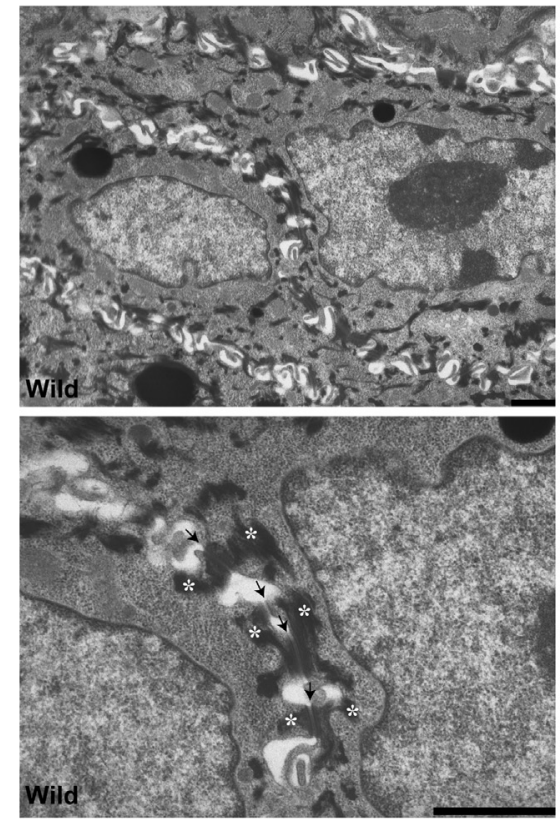

B

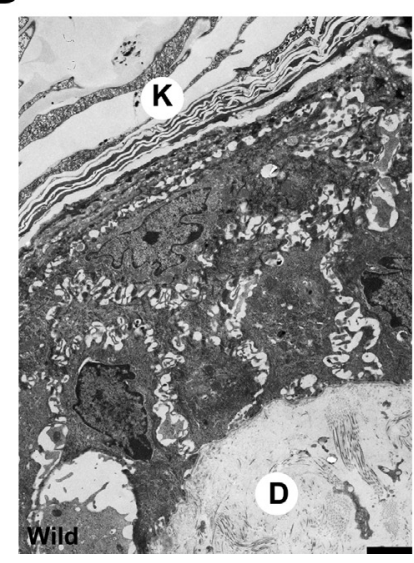

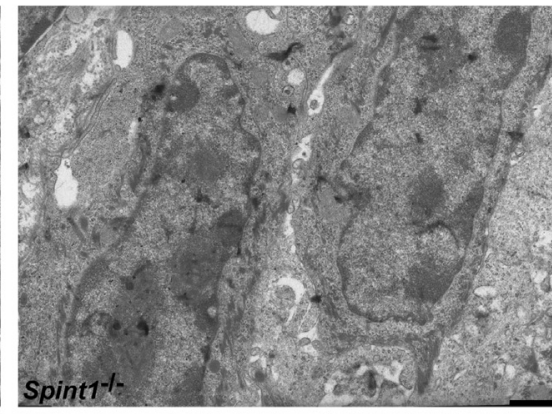

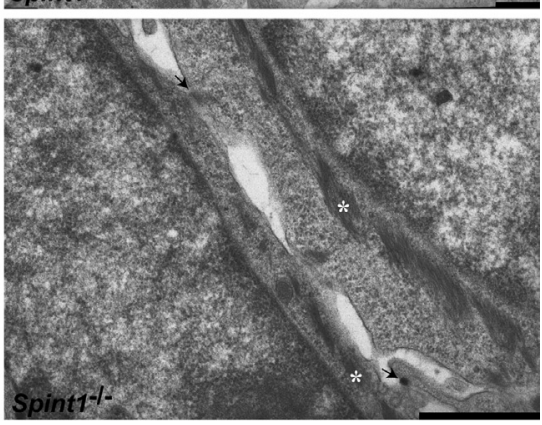

C

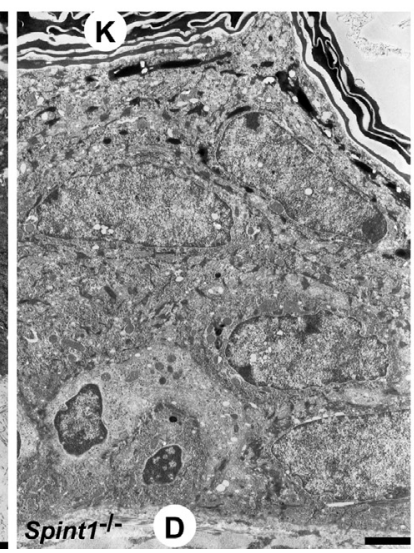

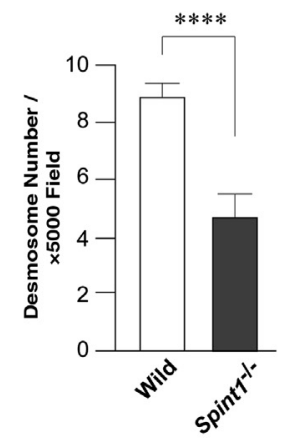

Figure 1 Transmission electron micrographs (TEMs) of epidermis from control C57BL/6 (wild) and hepatocyte growth factor activator inhibitor type 1 (HAI)-1-deficient (Spint1 ${ }^{-/-}$) mice. Tissues were analyzed from 7-day-old (A) and 14-dayold (B) mice. A: Intercellular digitation structure and assembly of keratin intermediate filaments (KIFs) to desmosomes are impaired in Spint $1^{-/-}$ mouse epidermis. Arrows indicate desmosomes. Representative KIFs are labeled by white asterisks. B: Loss of intercellular digitation structure is evident in Spint1 knockout mice. C: Decreased number of keratinocyte desmosomes in Spint $1^{-/-}$ epidermis. Mean of desmosome number in a $\times 5000$ TEM field was compared between wild-type epidermis and Spint $1^{-/-}$epidermis. Data are given as means \pm SEM (C). ${ }^{* * * * P}<0.0001$. Scale bars: $500 \mathrm{~nm}$ (A); $2 \mu \mathrm{m}$ (B). D, dermis; K, keratin layer. 
dimethyl sulfoxide; final concentration, $10 \mu \mathrm{mol} / \mathrm{L})$ or vehicle (dimethyl sulfoxide) was added into the medium.

\section{TEM}

For transmission electron microscopy (TEM), specimens were fixed with a mixture of $2 \%$ paraformaldehyde and $2.5 \%$ glutaraldehyde in $0.1 \mathrm{~mol} / \mathrm{L}$ phosphate buffer, $\mathrm{pH} 7.4$. After rinsing with the buffer, the specimens were post-fixed with $1 \%$ osmium tetroxide in $0.1 \mathrm{~mol} / \mathrm{L}$ phosphate buffer for 2 hours at $4{ }^{\circ} \mathrm{C}$. Post-fixed specimens were washed with distilled water, and then dehydrated through a graded ethanol series, substituted with propylene oxide, and embedded in epoxy resin. Ultrathin sections (80 $\mathrm{nm}$ thick) were cut and stained with $2 \%$ uranyl acetate in $70 \%$ methanol for 4 minutes, followed by Reynolds' lead citrate for 3 minutes, and observed in an HT7700 TEM instrument (Hitachi High-Technologies, Tokyo, Japan). To count the desmosome number, electron microscopic images that included desmosomal plaques within both keratinocytes were taken at $\times 5000$, and $>10$ fields were examined. ${ }^{33}$

\section{Gene Silencing and Overexpression}

To establish a subline of HaCaT cells in which HAI-1 was stably knocked down, shRNA against HAI-1 was inserted
A

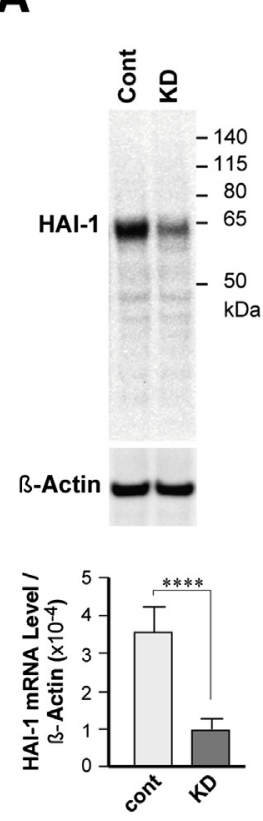

D

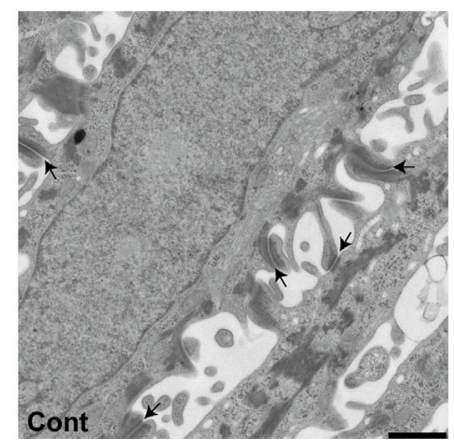

B

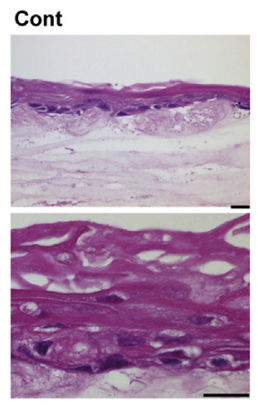

KD

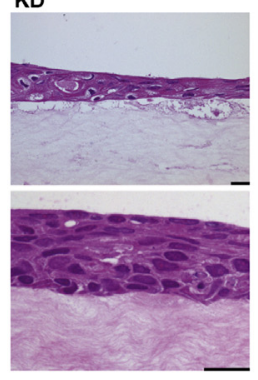

C
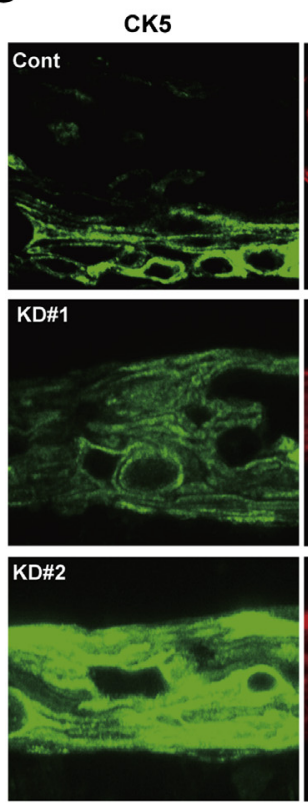
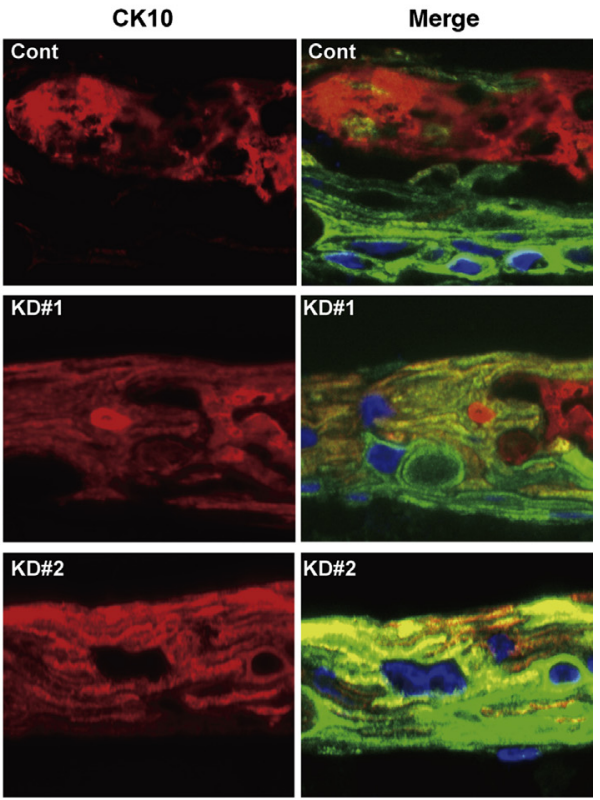

E
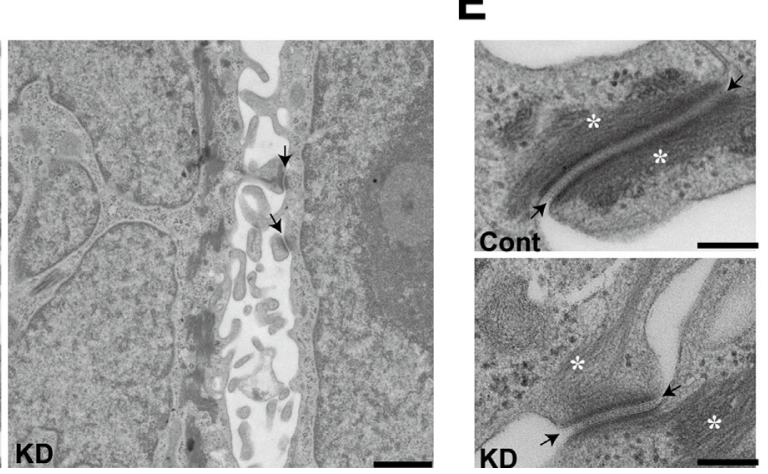

$\mathbf{F}$

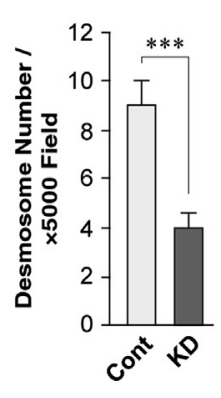

Figure 2 Desmosome and keratin intermediate filament (KIF) assembly into desmosomes decreases in three-dimensional (3D) organotypic culture of hepatocyte growth factor activator inhibitor type 1 (HAI)-1 knockdown (KD) HaCaT cells. A: Efficiency of stable HAI-1 KD by shRNA lentiviral vector in HaCaT cells. Immunoblotting and quantitative real-time RT-PCR data for HAI-1. B: Altered morphology of 3D organotypic culture of HAI-1 KD HaCaT cells (KD) compared with control HaCaT cells (Cont). Hematoxylin and eosin stain. C: Expression patterns of cytokeratin (CK) 5 and CK10 in 3D organotypic culture. Polarity of the cytokeratin expression is well preserved in control HaCaT (Cont) 3D culture, but not in HAI-1 KD HaCaT 3D culture. For HAI-1 KD cells, data of two separated experiments (KD 1 and KD 2) are shown. D and E: Transmission electron micrograph of 3D organotypic culture. Keratin intermediate filaments (KIF) assembly into desmosomes is decreased in HAI-1 KD cells. Arrows indicate desmosomes. Representative KIFs are labeled by white asterisks. F: Effects of HAI-1 KD on desmosome number. ${ }^{* * *} P<0.001,{ }^{* * * * P}<0.0001 . n=3$ (A). Scale bars: $20 \mu \mathrm{m}$ (B); $1 \mu \mathrm{m}$ (D); $200 \mathrm{~nm}$ (E). 
into pLenti4/BLOCK-iT (Life Technologies Japan). Generation of the lentiviral particles and transfection into $\mathrm{HaCaT}$ cells were performed according to the manufacturer's instructions. Then, phleomycin D1 (Zeocin, Life Technologies Japan)-resistant stable HAI-1 KD cells were obtained.

For transient KD of matriptase and prostasin, siRNAs (Stealth RNAi; Life Technologies Japan) were used. The sequences were as follows: 5'-GCGUGUACACAAGGCUCCCUCUGUU-3' (human matriptase siRNA 1), 5'-GCUUGCUGGUGUGGCACUUCCAUUA-3' (mouse matriptase siRNA), and 5'-GGCCAUUCUGCUCUAUCUUGGAUUA-3' (human prostasin siRNA). Stealth RNAi Negative Control Duplexes (Invitrogen) were transfected as a control. For human matriptase, another siRNA sequence (human matriptase siRNA 2) (Qiagen, Valencia, CA), 5'-CGUCGUCACUUGUACACCAdTdT-3', was also used with scrambled siRNA as a control. For transient KD of PAR-2, siRNA pool (ON-TARGETplus SMARTpool siRNA; Thermo Scientific, Yokohama, Japan) was used with siGENOME Non-Targeting siRNA pool as a control. Transfection was performed using Lipofectamine 2000 reagent (Invitrogen), followed by cultivation in DMEM supplemented with $10 \%$ FBS for 24 hours. Then, the cells were washed with phosphate-buffered saline (PBS) and maintained in the serum-free DMEM. At 72 hours after the transfection, cells were harvested and proteins were extracted.

For engineered overexpression of HAI-1, mouse or human HAI-1 cDNA was subcloned into pLenti6.3/TO/V5 (Invitrogen). Generation of the lentiviral particles and transfection into primary keratinocytes from Spint $1^{-/-}$mice or HAI-1 KD HaCaT cells were performed according to the manufacturer's instructions.

\section{Quantitative RT-PCR}

Total RNA was prepared with TRIzol (Life Technologies), followed by DNase I (Takara Bio, Shiga, Japan) treatment. For RT-PCR, total RNA ( $3 \mu \mathrm{g}$ ) was reverse transcribed with a mixture of oligo (dT)12-18 (Life Technologies) and random primers (6-mer) (Takara Bio) using $200 \mathrm{U}$ of ReverTra Ace (TOYOBO, Osaka, Japan). The primer sequences are described in Table 1. For quantitative RT-PCR, PCR was performed in a Thermal Cycler Dice Real Time System II (Takara Bio) using the SYBR Premix Ex Taq II (Takara Bio). For internal control, $\beta$-actin mRNA was also measured.

\section{Immunohistochemical and Immunocytochemical Analyses}

Skin tissues of Spint1 ${ }^{-/-}$and wild-type C57BL/6 mice were fixed in $4 \%$ paraformaldehyde in PBS overnight and then dehydrated and embedded in paraffin. For immunohistochemistry, tissue sections were processed for antigen retrieval by microwaving for 10 minutes at $96^{\circ} \mathrm{C}$ in 10 $\mathrm{mmol} / \mathrm{L}$ citrate buffer ( $\mathrm{pH} 6.0$ ), followed by treatment with $3 \% \mathrm{H}_{2} \mathrm{O}_{2}$ in PBS for 10 minutes. After blocking in 5\% normal goat serum (Dako) in PBS, the sections were incubated with primary antibody for 16 hours at $4^{\circ} \mathrm{C}$ and then incubated with Envision-labeled polymer reagents (Dako) for 30 minutes at room temperature. The reactions were revealed by nickel, cobalt-3,3'-diaminobenzidine (Pierce, Rockford, IL), and counterstained with Mayer's hematoxylin. For immunocytochemistry, cultured cells were fixed with $4 \%$ paraformaldehyde in PBS for 15 minutes, followed by blocking for 60 minutes with $5 \%$ normal goat serum (Dako) at room temperature. Then, the cells were incubated with primary antibody for 16 hours at $4^{\circ} \mathrm{C}$. After washing, the cells were incubated for 20 minutes at room temperature with Alexa Fluor 488-conjugated goat anti-rabbit IgG (Invitrogen) for phosphorylated p38 and CK5, Alexa Fluor 594-conjugated goat anti-mouse IgG (Invitrogen) for CK10, or Alexa Fluor 488-conjugated goat anti-mouse IgG (Invitrogen) for matriptase (M24 mAb). In an indicated experiment, the cells were washed and further incubated with Alexa Fluor 488-conjugated phalloidin (Invitrogen) for 20 minutes at room temperature. Then, the cells were counterstained with DAPI (Sigma), and examined with an Axio Imager A2 (Carl Zeiss MicroImaging, Tokyo, Japan).
A

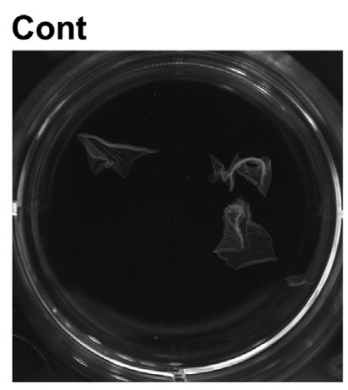

KD

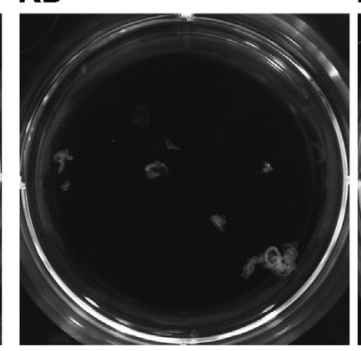

KD

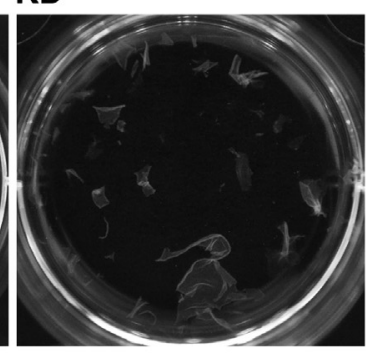

B

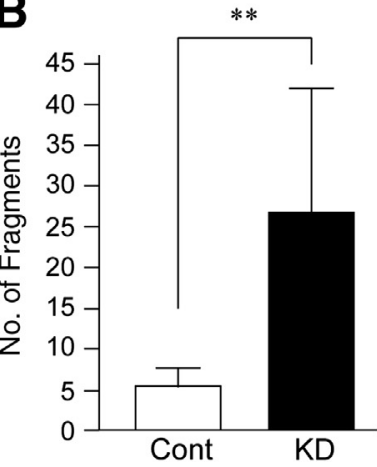

Figure 3 Enhanced vulnerability to mechanical shear stress in hepatocyte growth factor activator inhibitor type 1 (HAI)-1 knockdown (KD) HaCaT. A: Representative images of control (Cont) and HAI-1 KD (KD) HaCaT cells after dispase mechanical dissociation assay. B: Number of fragments generated by mechanical shear stress in the dispase assay. Data are given as means $\pm \mathrm{SD}(\mathbf{B}) .{ }^{* *} P<0.01$. 


\section{Immunoblot Analysis}

Cellular proteins were extracted with $1 \%$ Triton X-100 (Nacalai Tesque, Kyoto, Japan) in PBS and centrifuged at $10,000 \times g$ for 15 minutes, and the supernatants (ie, Triton $\mathrm{X}-100$ soluble fraction) and the pellets (Triton X-100 insoluble fraction) were separately collected. For proteins in culture supernatant, cultured conditioned media were concentrated 10-fold with an Amicon-Ultra-4 (mol. wt. cutoff, $10 \mathrm{kDa}$; Millipore) and protein concentration was determined by the Bradford method (BioRad, Hercules,
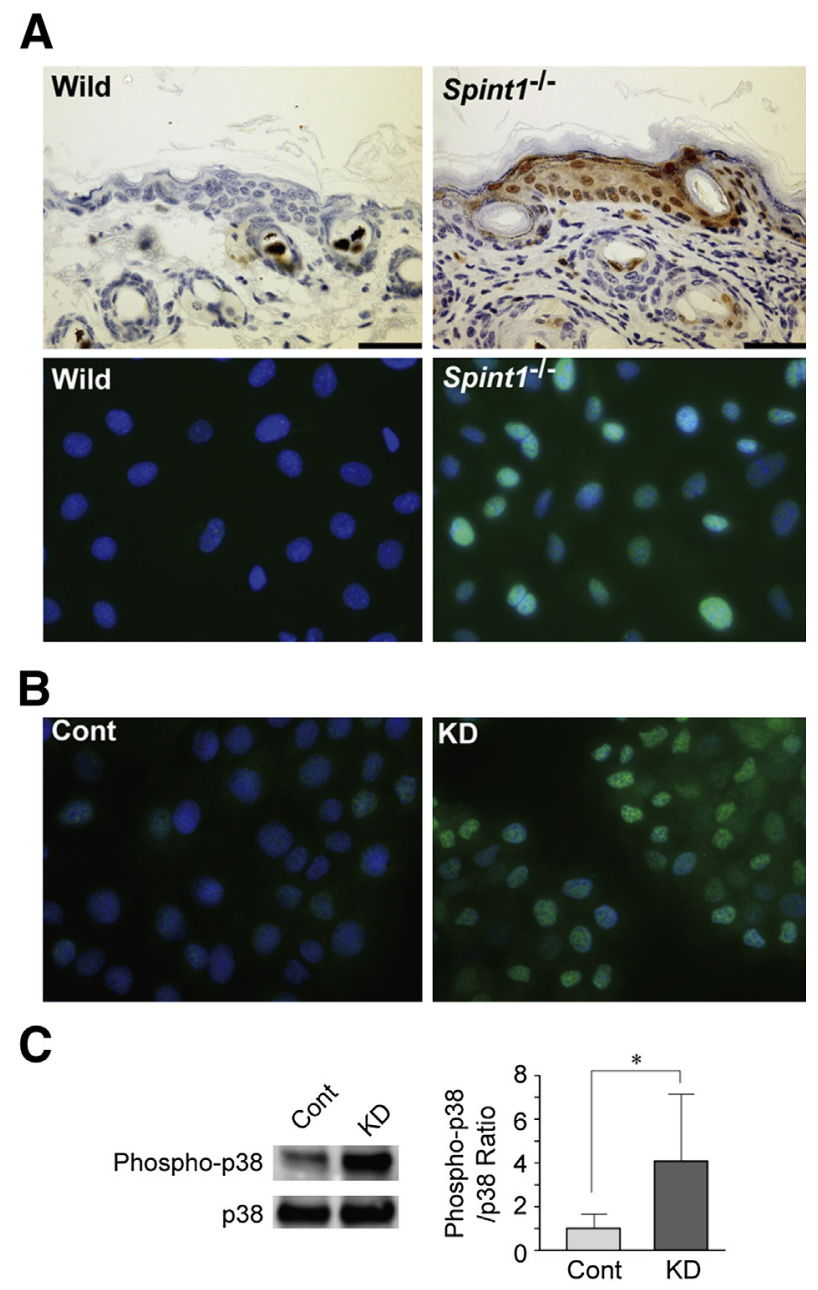

D

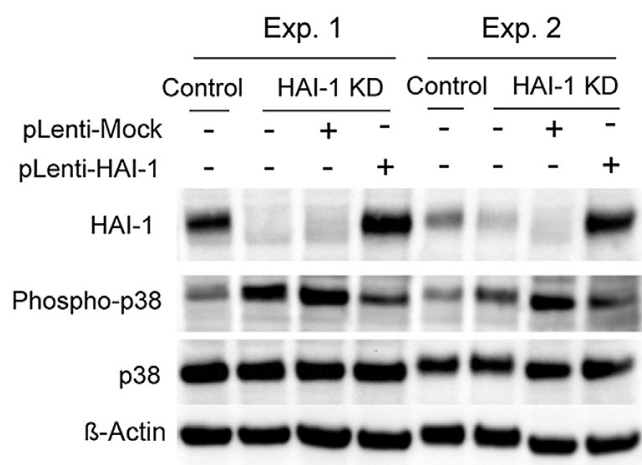

CA). Samples were separated by SDS-PAGE under nonreducing (for M24 and M69) or reducing (for other antibodies) conditions using $4 \%$ to $12 \%$ gradient gels (Invitrogen) and transferred onto an Immobilon membrane (Millipore). After blocking with 5\% nonfat dry milk in Tris-buffered saline with $0.05 \%$ Tween 20 , the membranes were incubated with primary antibodies at $4{ }^{\circ} \mathrm{C}$ overnight, followed by washing with Tris-buffered saline with $0.05 \%$ Tween 20 and incubation with horseradish peroxidase-conjugated secondary antibodies (Dako) diluted in Tris-buffered saline with $0.05 \%$ Tween 20 with $1 \%$ bovine serum albumin for 1 hour at room temperature. The labeled proteins were visualized with a chemiluminescence reagent (PerkinElmer Life Science, Boston, MA).

\section{Dispase Mechanical Dissociation Assay}

Vulnerability of cultured epithelial layer to mechanical shear stress was assessed by a dispase mechanical dissociation assay, described previously. ${ }^{34}$ In brief, HaCaT cells were seeded in 6-well plates. After reaching confluency, cells were washed twice with PBS and then incubated with $2 \mathrm{~mL}$ of dispase II (2.4 U/mL DMEM; Sigma) for 30 minutes to detach the monolayer from the bottom, and the detached monolayer was transferred to a $15-\mathrm{mL}$ polypropylene centrifuge tube. Then, mechanical stress was applied by 50 inversions in $2 \mathrm{~mL}$ PBS and fragments were counted.

\section{Protease Activity Assay}

Conditioned medium from $\mathrm{HaCaT}$ cells was concentrated (10-fold) with an Amicon-Ultra-4 (Millipore) unit. Then, fluorogenic substrate Boc-Gln-Ala-Arg-AMC (Peptide Institute, Inc., Osaka, Japan) was added to a final concentration of $10 \mu \mathrm{mol} / \mathrm{L}$, fluorescence release was measured using a FlexStation 3 (Molecular Devices, Tokyo, Japan), and the activity was expressed in terms of the velocity maximum value $\left(V_{\max }\right)$. The concentrated conditioned

Figure 4 Enhanced activation of p38 in response to hepatocyte growth factor activator inhibitor type 1 (HAI-1) insufficiency in keratinocytes. A: Immunohistochemistry of mouse epidermis (top panels) and immunocytochemistry of primary cultured keratinocytes (bottom panels) for phosphorylated p38. Enhanced nuclear immunoreactivity is noted in HAI-1-deficient (Spint1 K0) mouse keratinocytes compared with control C57BL/6 (wild) mouse keratinocytes. B: Phosphorylated p38 immunocytochemistry of $\mathrm{HaCaT}$ cells in two-dimensional culture, showing enhanced nuclear immunoreactivity in HAI-1 knockdown (KD) HaCaT cells (KD) compared with control HaCaT cells (Cont). C: Immunoblot for phosphorylated p38 in control and HAI-1 KD HaCaT cells. Cultured cells were washed with phosphate-buffered saline and maintained in serum-free Dulbecco's modified Eagle's medium for 24 hours before extraction. Ratio of phosphorylated $p 38$ signal/total $p 38$ signal was calculated and plotted. D: Effect of HAI-1 reversion on HAI-1 KD-induced enhanced p38 phosphorylation in $\mathrm{HaCaT}$ cells. Immunoblot results of two separate experiments (Exp.) are shown. Forced expression of HAI-1 suppresses p38 phosphorylation induced by shRNA-mediated silencing of HAI-1. Data are given as means \pm SD (C). $n=4$ (C). ${ }^{*} P<0.05$. Scale bar $=50 \mu \mathrm{m}$. 
media were also used for gelatin zymography to detect activated matriptase. For gelatin zymography, samples were electrophoresed in 10\% SDS-polyacrylamide gels impregnated with $1 \mathrm{mg} / \mathrm{mL}$ gelatin (KOKEN Co.) under nonreducing conditions, as described previously. ${ }^{22}$ After electrophoresis, gels were washed in $50 \mathrm{mmol} / \mathrm{L}$ Tris- $\mathrm{HCl}$ (pH 7.5) containing $0.1 \mathrm{~mol} / \mathrm{L} \mathrm{NaCl}$ and $2.5 \%$ Triton $\mathrm{X}$ 100 for 1.5 hours to remove SDS, followed by incubation in $50 \mathrm{mmol} / \mathrm{L}$ Tris- $\mathrm{HCl}$ at $\mathrm{pH} 7.5$ and $0.5 \mathrm{mmol} / \mathrm{L}$ EDTA containing $10 \mu \mathrm{mol} / \mathrm{L}$ broad-spectrum metalloprotease inhibitor, GM6001 (Millipore), for 30 minutes to eliminate matrix metalloproteinase activities. Then, the gels were incubated at $37^{\circ} \mathrm{C}$ for 72 hours in $50 \mathrm{mmol} / \mathrm{L}$ Tris- $\mathrm{HCl}(\mathrm{pH}$ 7.5), and stained with Coomassie Brilliant Blue.

\section{Statistical Analysis}

Statistical analysis was done using SPSS software version 15.0 (SPSS Japan Inc., Tokyo, Japan). Comparisons between two paired groups or two unpaired groups were made with the Wilcoxon signed rank test or $U$-test, respectively. Significance was set at $P<0.05$.

\section{Results}

\section{HAI-1 Deficiency Leads to Decreased Convergence of KIF on Desmosomes in Keratinocytes}

We investigated morphological abnormalities of epidermal keratinocytes in dorsal skin TEM specimens taken from Spint1-deleted mice 7 and 14 days after birth. At low-magnification TEM, the intercellular spaces between keratinocytes were indistinct in the epidermis of Spint1-deleted mice compared to those of wild-type C57BL/6 mice (Figure 1, A and B). The intercellular spaces and intercellular bridges of normal epidermis observed in tissue sections reflect a shrinkage artifact because the keratinocytes pull away from each other during dehydration, and remain connected only by their well-developed abundant desmosomal junctions. The epithelial cells with less-developed desmosomal junctions shrink without pulling each other and do not form such structures. Therefore, we evaluated desmosomes of the keratinocytes in detail. Higher magnification revealed that the HAI-1-deficient epidermis possessed poorly developed intercellular digitations. Moreover, although desmosomes were formed, KIFs attached to the desmosomes were significantly reduced in HAI-1-deficient keratinocytes compared with wild-type keratinocytes (Figure 1A). Indeed, desmosomes significantly decreased in number in the Spint $1^{-1-}$ mouse epidermis compared to the wild-type epidermis (Figure 1C).

To test whether the above observed morphological changes caused by HAI- 1 deficiency were reproducible in human keratinocytes, we silenced HAI-1 in the $\mathrm{HaCaT}$ human keratinocyte line. Stable expression of
HAI-1-specific shRNA resulted in $66 \%$ suppression of the HAI-1 protein level (Figure 2A). Then, HAI-1 KD $\mathrm{HaCaT}$ cells and control vector-transfected $\mathrm{HaCaT}$ cells were used in a 3D organotypic culture. Although both HAI-1 KD and control HaCaT cells formed epidermislike multilayered structures, HAI-1 KD HaCaT showed a less differentiated morphology compared with control cells that showed an obvious tendency of keratinization in the upper layer (Figure 2B). Immunofluorescence analyses for CK5 and CK10 revealed that the polarity of cytokeratin expression is preserved in control HaCaT 3D culture and is disturbed in HAI-1 KD 3D culture (Figure 2C). Subsequent TEM analysis revealed a markedly decreased assembly of KIFs into desmosomes in HAI-1 KD cells, with a decreased number and size of desmosomes (Figure 2, D-F). Consequently, HAI-1 KD $\mathrm{HaCaT}$ cell sheets were more vulnerable to mechanical shear stress compared to control HaCaT (Figure 3).

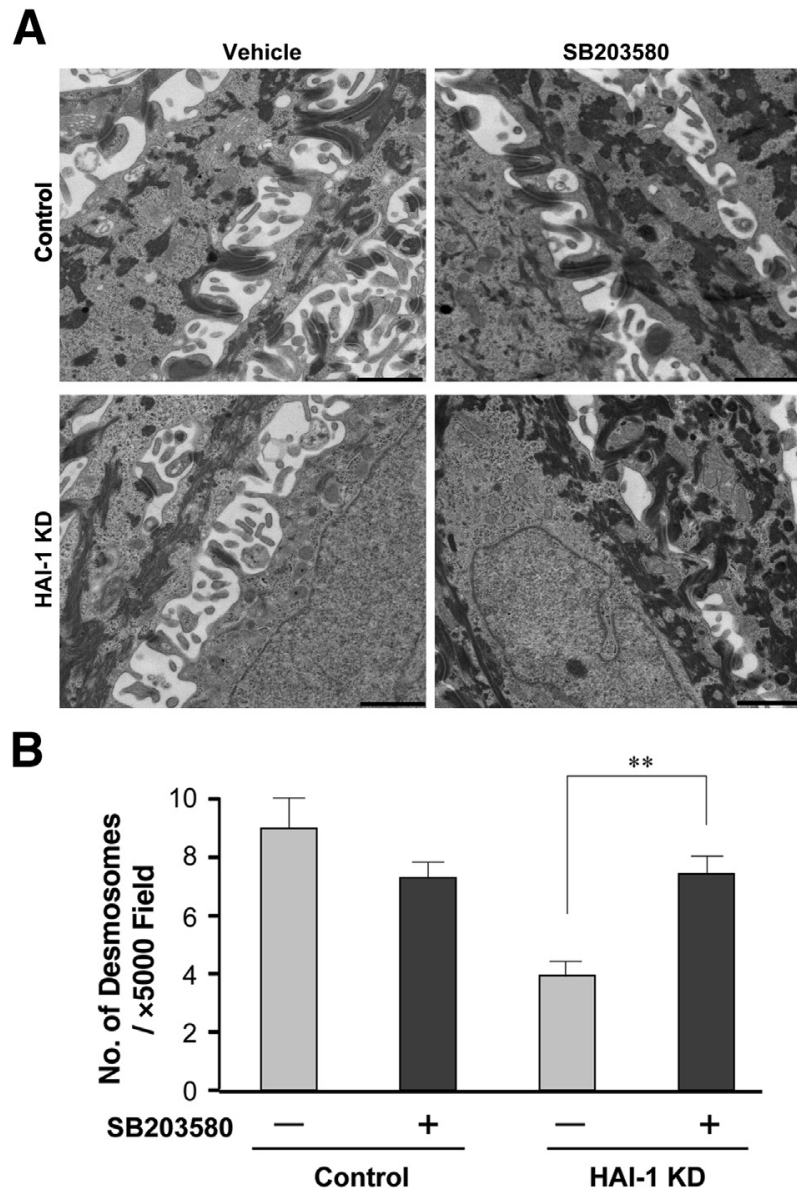

Figure 5 Effect of p38 inhibitor SB203580 on HaCaT three-dimensional organotypic culture. A: Transmission electron micrograph shows reversion of keratin intermediate filament assembly into desmosomes in hepatocyte growth factor activator inhibitor type 1 (HAI-1) knockdown (KD) HaCaT cells in response to the addition of $10 \mu \mathrm{mol} / \mathrm{L} \mathrm{SB203580} \mathrm{in} \mathrm{culture} \mathrm{me-}$ dium. B: Desmosome number recovers in HAI-1 KD HaCaT cells treated with SB203580. Data are given as means $\pm \mathrm{SD}(\mathbf{B}) . n=3$ (B). ${ }^{*} P<0.01$. Scale bar $=1 \mu \mathrm{m}$. 


\section{HAI-1-Deficient Keratinocytes Show Enhanced Activation of p38 MAPK}

We next investigated molecular mechanisms responsible for the decreased KIF aggregation into desmosomes in HAI$1-$ deficient keratinocytes. Because p38 MAPK activation has been reported to induce retraction of keratin filaments, ${ }^{6}$ we evaluated the nuclear translocation and phosphorylation of $\mathrm{p} 38$ in Spint1-deleted epidermis and HAI-1 KD HaCaT cells. Enhanced nuclear translocation of phosphorylated p38 was observed in Spint1-deleted epidermis, as well as in primary cultured keratinocytes from Spint 1-deleted mice, compared with wild-type controls (Figure 4A). Similar findings were obtained in HAI-1 KD HaCaT cells (Figure 4B), with increased levels of phosphorylated p38 in immunoblot analyses (Figure 4C). To confirm that the enhanced p38 phosphorylation was, in fact, caused by HAI-1 silencing, we then examined the effect of HAI1 reversion on HAI-1 KD HaCaT cells. The restoration of HAI-1 suppressed the p38 phosphorylation in HAI-1 KD cells (Figure 4D). These observations suggested that HAI-1 loss enhanced the activation of p38 MAPK in keratinocytes.

\section{Activation of p38 MAPK Is Responsible for HAI-1 Deficiency-Induced Impaired Assembly of KIF and Reduced Number of Desmosomes}

To determine whether the activation of p38 MAPK was associated with the abnormalities in HAI-1-deficient keratinocytes, we tested the effect of a specific p38 inhibitor, SB203580, on organotypic culture of HaCaT cells. The addition of SB203580 restored the density of KIFs that assembled into desmosomes in HAI-1 KD HaCaT cells (Figure 5A), accompanying the reversion of the desmosome number (Figure 5B). Treatment of SB203580 had no effect on control HaCaT cells. These results suggested that the activation of p38 MAPK was responsible for the ultrastructural abnormalities observed in HAI-1-deficient keratinocytes.

\section{Enhanced p38 MAPK Activation in HAI-1-Deficient Keratinocytes Is Mediated by PAR-2 Signaling}

We investigated how $\mathrm{p} 38$ MAPK was activated in HAI-1 KD $\mathrm{HaCaT}$ cells. Previous studies suggested that PAR-2 activates p38 MAPK signaling. ${ }^{35}$ PAR-2 is widely expressed in the skin and activated by trypsin-like proteases. ${ }^{27}$ Thus, we hypothesized that activation of p38 MAPK signaling in HAI-1 KD $\mathrm{HaCaT}$ cells may be caused by inadequate activity of trypsinlike serine protease(s) in the absence of sufficient HAI-1. To confirm this hypothesis, we examined the effect of a PAR-2 agonist (SLIGR) and an antagonist (FSLLRY) on the activation of $\mathrm{p} 38$ MAPK in HaCaT cells. Treatment with the PAR-2 agonist enhanced phosphorylation of p38 MAPK in HaCaT cells (Figure 6A), which was accompanied by increased nuclear translocation of p38 (Figure 6B). In contrast, PAR-2 antagonist abrogated the HAI-1 KD-induced enhanced phosphorylation of $\mathrm{p} 38$, but did not affect the basal phosphorylation

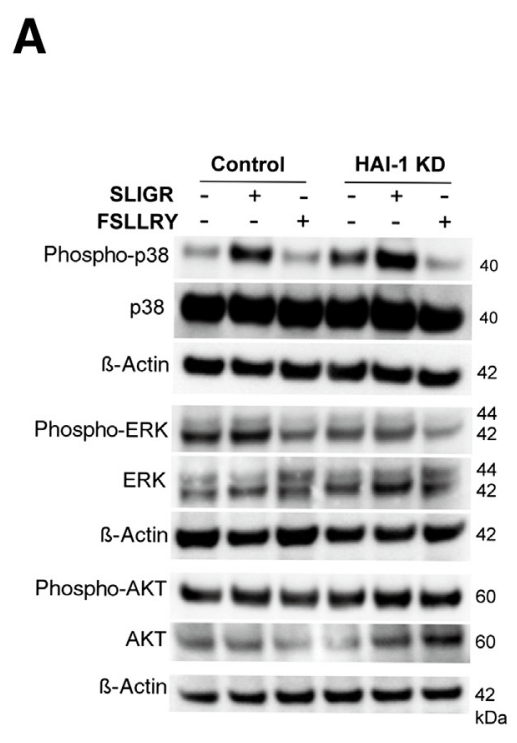

B
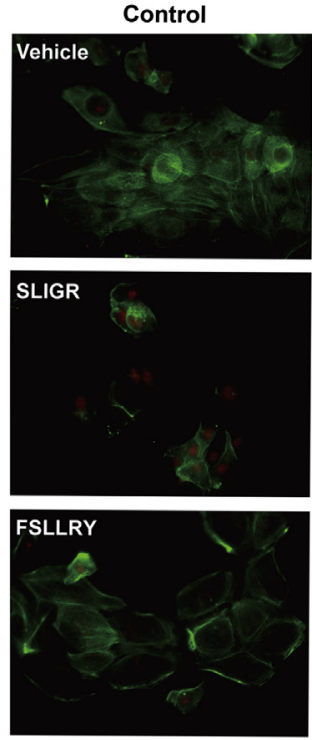

C
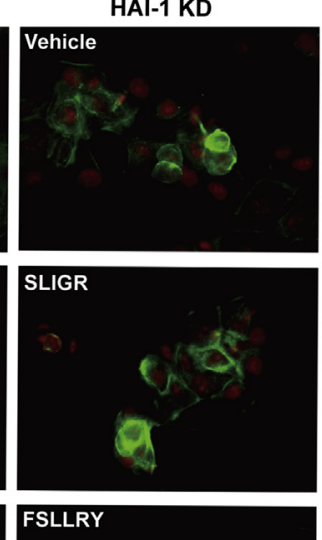

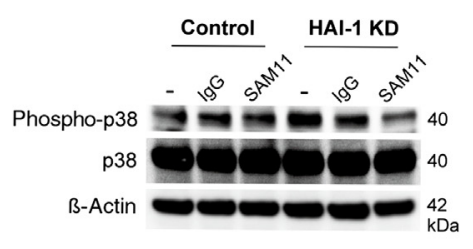

Figure 6 Activation of $\mathrm{p} 38$ by protease-activated receptor-2 (PAR-2) signaling in HaCaT cells. A: Effects of $100 \mu$ mol/L PAR-2 agonist (SLIGR) and $100 \mu \mathrm{mol} / \mathrm{L}$ antagonist (FSLLRY) peptides on phosphorylation of p38, extracellular signal-regulated kinase (ERK), and AKT in control and hepatocyte growth factor activator inhibitor type 1 (HAI-1) knockdown (KD) HaCaT cells. Cultured cells were washed with PBS and maintained in serum-free Dulbecco's modified Eagle's medium (DMEM) with or without the peptide for 24 hours before extraction. B: Immunocytochemical assessment of phosphorylated p38. Phosphorylated $\mathrm{p} 38$ and actin are indicated in red and green, respectively. Nuclear immunoreactivity of phosphorylated p38 was observed in response to HAI-1 KD or PAR-2 agonist, and HAI-1 KD-induced p38 activation was suppressed by PAR-2 antagonist. C: Effect of PAR-2 neutralizing antibody (SAM11) on phosphorylation of $\mathrm{p} 38$. The cells were cultured with or without $20 \mu \mathrm{g} / \mathrm{mL}$ of SAM11 in serum-free DMEM for 24 hours. 
level of p38 (Figure 6, A and B). Although ERK phosphorylation was modestly suppressed by the PAR-2 antagonist, it was not enhanced by the PAR-2 agonist or HAI-1 KD. Neither the PAR-2 agonist nor the antagonist altered the phosphorylation level of AKT, although there were modest changes of total AKT levels that are difficult to explain at present. To further confirm the role of PAR-2 in HAI-1 loss-induced p38 activation, we examined the effect of a PAR-2 neutralizing antibody (SAM11) on p38 activation. Treatment of HAI-1 KD $\mathrm{HaCaT}$ cells with SAM11 abolished enhanced p38 phosphorylation, but the basal level of phosphorylation of control cells was not affected by SAM11 (Figure 6C). These data indicated that the HAI-1 KD-induced activation of p38 MAPK was mediated by PAR-2 activation in $\mathrm{HaCaT}$ cells.

\section{Trypsin-Like Protease Activity Increases in HAI-1 KD HaCaT Culture Supernatant}

PAR-2 is activated by trypsin-like serine proteases, and matriptase is an efficient activator of PAR-2 in epithelial cells. ${ }^{17,27,30}$ The evidence suggested that the most important cognate protease of HAI-1 in keratinocytes is matriptase. ${ }^{24}$ Therefore, we hypothesized that the activity of matriptase was enhanced because of insufficient HAI-1, which eventually led to PAR-2 activation in HAI KD HaCaT cells. To test this hypothesis, we examined cellular expression of matriptase and its activity in culture supernatants of $\mathrm{HaCaT}$ cells. Quantitative real-time RT-PCR showed that the matriptase mRNA level was slightly decreased by the suppression of HAI-1 expression in $\mathrm{HaCaT}$ cells, but the difference was not statistically significant (Figure 7A). In cellular extracts from 3D culture of control HaCaT cells, 120-kDa matriptaseHAI-1 complex was detected (Figure 7B), which was also labeled by anti-activated matriptase M69 mAb (Figure 7C). In contrast, this complex was hardly detectable in extracts of two-dimensional culture, and the levels of total cellular matriptase labeled by M24 mAb were comparable between HAI-1 KD and control HaCaT cells (Figure 7C). Those matriptase bands corresponded to promatriptase, because they were barely detectable with M69 mAb. However, when

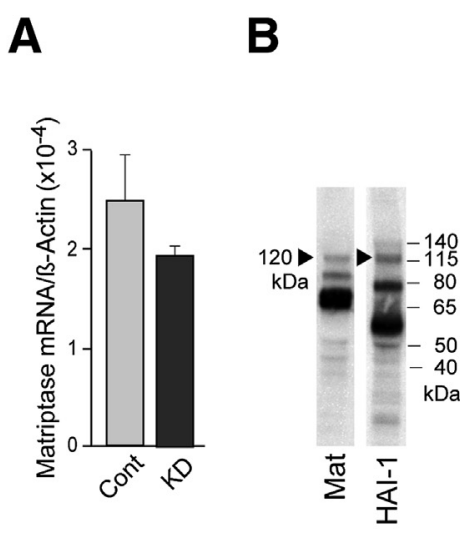

D

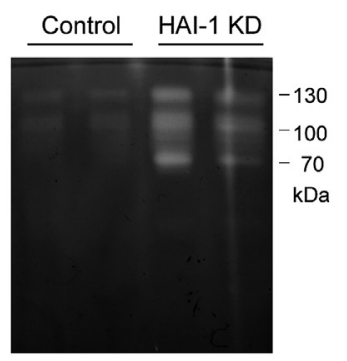

C

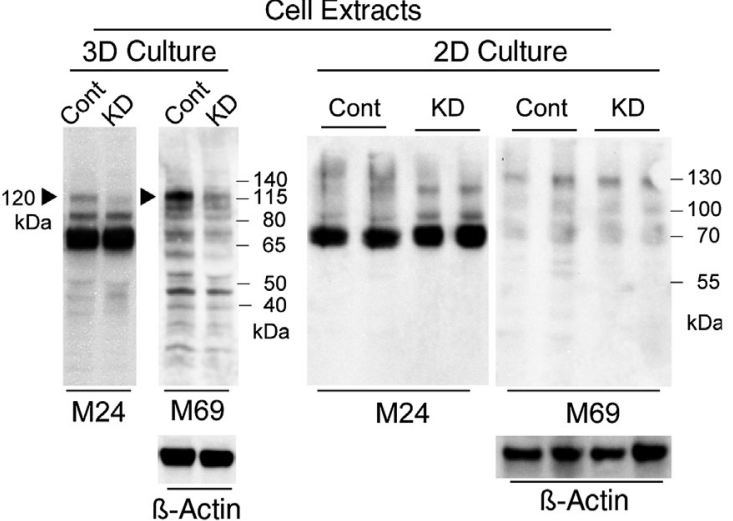

$\mathbf{F}$

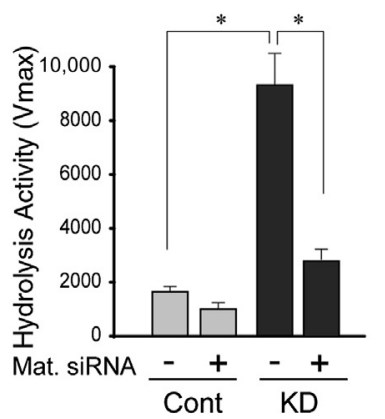

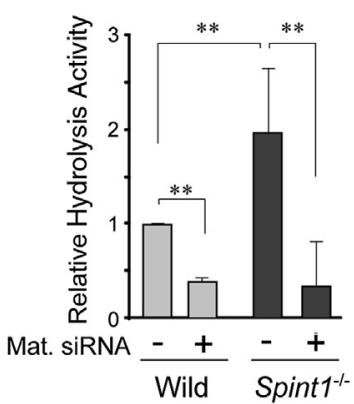

G

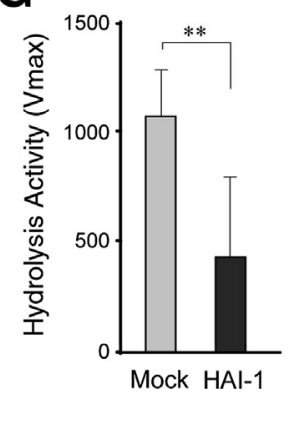

Figure 7 Enhancement of matriptase activity by hepatocyte growth factor activator inhibitor type 1 (HAI-1) knockdown (KD). A: Matriptase mRNA level in control (Cont) and HAI-1 KD (KD) HaCaT cells. Data from real-time RT-PCR are indicated. B: Matriptase (Mat)-HAI-1 complex in HaCaT three-dimensional (3D) organotypic culture. Immunoblot data of cellular extracts from HaCaT 3D culture for Mat (M24 antibody) and HAI-1. Arrowhead indicates a 120-kDa matriptase-HAI-1 complex band. C: Immunoblot analyses of matriptase in cell extracts of 3D culture and two-dimensional (2D) culture, and serum-free culture media supernatants of 2D culture. Anti-total matriptase (M24) and anti-activated matriptase (M69) were used. D: Gelatin zymography of culture medium supernatant in the presence of metalloprotease inhibitor GM6001. Two independent samples from each control or HAI-1 KD HaCaT cells were analyzed. E: Boc-Gln-Ala-Arg-AMC hydrolysis activity $\left(V_{\max }\right)$ in culture supernatants of control and HAI-1 KD HaCaT with or without matriptase KD by matriptase siRNA 1. F: Boc-Gln-Ala-Arg-AMC hydrolysis activity of culture supernatant of epidermal keratinocytes from wild-type or Spint $1^{-/-}$mice with or without matriptase KD. Values are relative $V_{\max }$ to wild-type keratinocyte supernatant. G: Effect of forced mouse HAI-1 expression on Boc-Gln-Ala-Arg-AMC hydrolysis activity in Spint $1^{-/-}$keratinocyte supernatant. Data are given as means \pm SD (A). $n=3(\mathbf{A}$ and $\mathbf{G}) ; n=5(\mathbf{F}) .{ }^{*} P<0.05,{ }^{* *} P<0.01$. 
immunoblot analyses were performed using culture supernatants, significantly enhanced matriptase signals were detectable in HAI-1 KD supernatants compared with control supernatants, and the M69-positive activated form of matriptase species $>100 \mathrm{kDa}$ was also increased (Figure 7C). These M69-positive matriptase bands in the supernatant were not labeled by anti-HAI-1 antibody (data not shown), and the precise molecular nature of these activated matriptase bands remains to be determined. Consistent with these observations, enhanced gelatinolytic activity was detected in conditioned medium from HAI-1 KD cells compared to that from control $\mathrm{HaCaT}$ cells, as assessed by zymography in the presence of matrix metalloproteinase inhibitor GM6001 (Figure 7D).

Subsequent analysis of the hydrolysis of the synthetic fluorogenic substrate Boc-Gln-Ala-Arg-AMC revealed enhanced activity of trypsin-like protease in HAI-1 KD conditioned medium, which was significantly suppressed by $\mathrm{KD}$ of matriptase by siRNA (Figure 7E and Supplemental Figure S1). In primary cultured mouse keratinocytes, matriptase KD partially alleviated the hydrolysis activity in the culture supernatant of Spintl $^{-1-}$ keratinocytes (Figure 7F). Consequently, forced expression of HAI-1 in
Spint $^{-1-}$ keratinocytes suppressed the Boc-Gln-Ala-ArgAMC hydrolysis activity (Figure $7 \mathrm{G}$ ). Taken together, these results showed that insufficient HAI-1 expression resulted in enhanced trypsin-like protease activity in keratinocytes, a part of which is derived from matriptase.

\section{PAR-2 Activation Reduces KIF Assembly and Decreases Desmosome Number in HAI-1 KD HaCaT Cells}

Next, we asked whether enhanced matriptase activity was involved in the activation of p38 MAPK in HAI-1 KD HaCaT cells. Thus, we examined the effect of matriptase KD on p38 activation in the cells. Transient transfection of matriptase siRNA 1 or 2 resulted in an efficient KD effect and suppressed the enhanced activation of $\mathrm{p} 38$ at least partly (Figure 8A). Transfection of matriptase siRNA 1 suppressed the HAI-1 KD-induced enhanced p38 activation by 53\% (Figure 8B). Notably, p38 activation was also alleviated to a similar extent (69\% inhibition) by $\mathrm{KD}$ of prostasin (Figure 8, A and B). Double KD of matriptase and prostasin suppressed the HAI-1 KD-induced p38 activation by $86 \%$ (Figure 8B and Supplemental Figure S2). On the other hand, KD of PAR-2 completely abolished the HAI-1 KD-induced

A

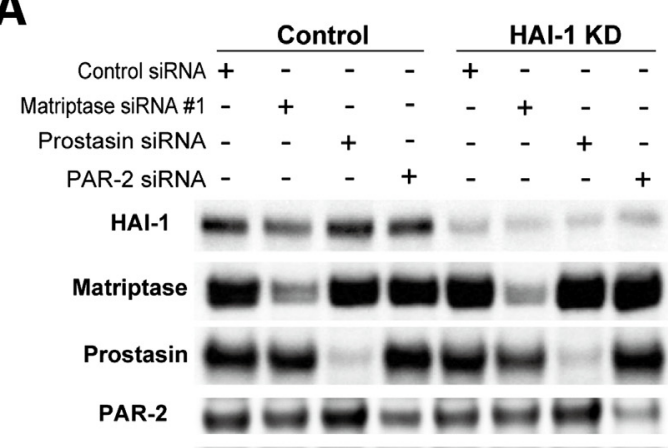

Phospho-p38 p38

B-Actin

B

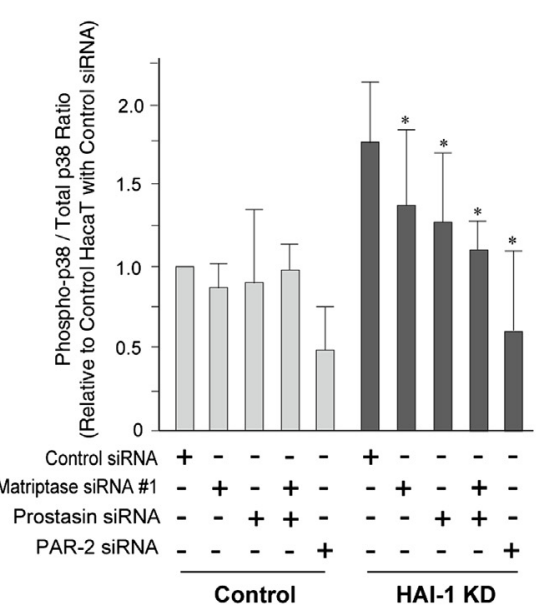

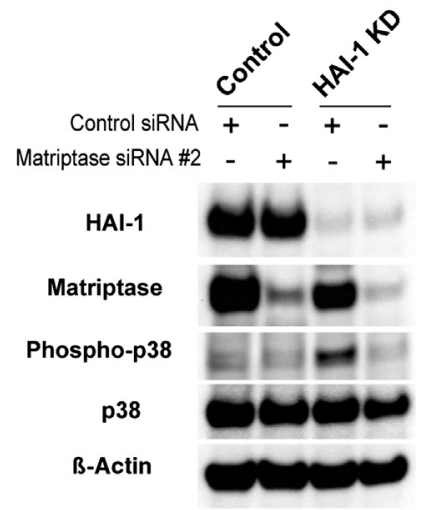

C

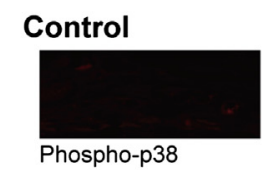

HAI-1 KD

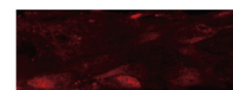

Phospho-p38

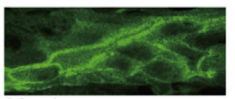

Matriptase

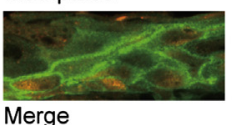

Figure 8 Effect of matriptase knockdown (KD), prostasin $K D$, matriptase/prostasin double $K D$, or protease-activated receptor-2 (PAR-2) KD on phosphorylation of p38 in control or hepatocyte growth factor activator inhibitor type 1 (HAI-1) KD $\mathrm{HaCaT}$ cells. A: Representative immunoblot data. For matriptase, another siRNA (siRNA 2) was also used to confirm the specific effect of matriptase KD on p38 phosphorylation. B: Relative ratio of phosphorylated p38/total p38. C: Immunocytochemistry of phosphorylated p38 in 3D organotypic culture. Enhanced p38 phosphorylation (red) is observed in HAI-1 KD HaCaT cells, and matriptase (green; M24 monoclonal antibody) is also expressed in these cells. Data are given as means $\pm \mathrm{SD}(\mathbf{B}) .{ }^{*} P<0.05$ compared to HAI-1KD HaCaT control siRNA. 
enhanced activation of p38 (Figure 8, A and B). The enhanced activation of p38 in HAI-1 KD HaCaT cells was also confirmed in 3D organotypic culture, and matriptase was also expressed in these cells (Figure 8C).

We also analyzed whether enhanced protease activity due to HAI-1 insufficiency did, in fact, impair the convergence of KIF and decrease the desmosome number in $\mathrm{HaCaT}$ cells through PAR-2 activation. First, we analyzed the effect of a PAR-2 agonist or an exogenous matriptase on control $\mathrm{HaCaT}$ cells in organotypic culture. Treatment with a PAR-2 agonist during organotypic culture decreased the number of desmosomes (Figure 9A) and KIF assembly (Supplemental Figure S3) in control HaCaT cells, and similar effects were induced by the addition of recombinant matriptase catalytic domain $(5 \mathrm{nmol} / \mathrm{L})$. Second, we examined the effect of a PAR-2 antagonist or a serine protease inhibitor (aprotinin) on the desmosome number in HAI-1 KD HaCaT cells. The PAR-2 antagonist, aprotinin, and the p38 inhibitor, SB203580, were capable of restoring the desmosome number (Figure 9A) and KIF assembly (Supplemental Figure S3). This finding was also supported, at least partly, by immunoblot analysis of desmoglein 3 , a desmosomal protein, even in two-dimensional cultured HaCaT cell extracts (Figure 9B). Immunoreactivity of desmoglein 3 was also decreased in 3D-cultured $\mathrm{HaCaT}$ cells in response to HAI-1 KD, whereas its mRNA level was rather preserved (Supplemental Figure S4).

\section{Discussion}

Herein, by using both in vitro and in vivo models, we showed, for the first time, that HAI-1 is required for KIF convergence toward desmosomes in epidermal keratinocytes. Insufficient HAI-1 in epidermal keratinocytes triggered augmented activation of PAR-2, followed by PAR-2-mediated p38 activation that eventually resulted in KIF retraction and decreased desmosome number. Our data also suggested that HAI-1 loss-induced activation of PAR-2 was mediated by an imbalance in cellular serine proteases and HAI-1.

It has become clear in recent years that serine proteases have important roles in epidermal homeostasis. For example, matriptase and prostasin are directly or indirectly implicated in profilaggrin processing, leading to epidermal barrier formation and protection against dehydration. Indeed, mice lacking St14 (matriptase) or Prss8 (prostasin) genes showed a severe defect in epidermal barrier function and died from dehydration early in neonatal development. ${ }^{36,37}$ In humans, amino acid substitutions in the highly conserved protease domain of matriptase result in reduction or loss of matriptase activity and cause autosomal recessive ichthyosis with hypotrichosis. ${ }^{38}$ On the other hand, excess expression or activity of serine protease is associated with skin disorders. For example, transgenic expression of prostasin in mouse skin showed scaly skin reminiscent of ichthyosis, epidermal hyperplasia, disrupted skin barrier function, and inflamed skin. ${ }^{31}$ Transgenic

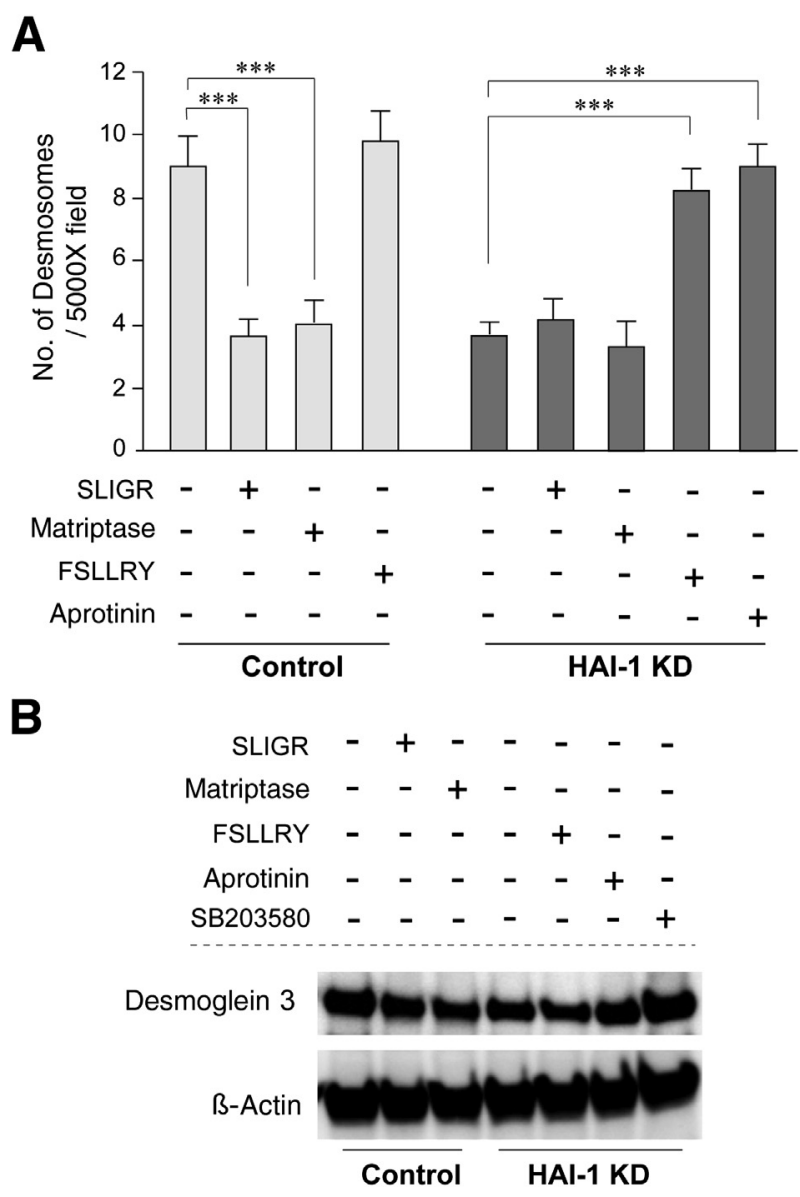

Figure 9 Involvement of protease-mediated protease-activated receptor-2 (PAR-2) signaling on desmosome number. A: Effects of PAR-2 agonist (SLIGR) or antagonist (FSLLRY), matriptase, or aprotinin on desmosome number in three-dimensional organotypic culture of control and hepatocyte growth factor activator inhibitor type 1 (HAI-1) knockdown (KD) HaCaT cells. B: Effects of $100 \mu \mathrm{mol} / \mathrm{L}$ PAR-2 agonist or $100 \mu \mathrm{mol} / \mathrm{L}$ antagonist, $5 \mathrm{nmol} / \mathrm{L}$ matriptase, $200 \mu \mathrm{g} / \mathrm{mL}$ aprotinin, or $10 \mu \mathrm{mol} / \mathrm{L} \mathrm{p} 38$ inhibitor (SB203580) on desmoglein 3 protein level in cultured HaCaT cells. Cultured cells were washed with phosphate-buffered saline and maintained in serum-free Dulbecco's modified Eagle's medium with or without reagent for 24 hours before extraction. Triton X-100 insoluble fraction of the cellular extract was used for immunoblotting. ${ }^{* \star *} P<0.001$.

expression of matriptase in mouse keratinocytes resulted in skin carcinogenesis that could be suppressed by concomitant overexpression of HAI- $1 .{ }^{39}$ Excess activity of KLK-5, caused by insufficient serine protease inhibitor of Kazal type 5, results in Netherton syndrome, a rare autosomal recessive skin disease with severe skin inflammation, scaling, hair shaft defects, and allergic manifestations. ${ }^{40}$ Recent studies have suggested that PAR-2 is crucially involved in these protease-mediated skin abnormalities. ${ }^{27,30,31}$

PAR-2 is expressed by keratinocytes and plays an important physiological role in the epidermis. Abnormal expression or activity of PAR-2 has been associated with several inflammatory skin disorders involving barrier abnormalities, such as atopic dermatitis, Netherton syndrome, psoriasis, and peeling skin syndrome. ${ }^{27,40-42}$ Moreover, abnormal skin 
phenotypes observed in prostasin and matriptase transgenic/ mutant mice were completely abrogated when the $F 2 r l l$ gene encoding PAR-2 was deleted concomitantly. ${ }^{30,31}$ Therefore, regulation of PAR-2 signaling is critically important for epidermal homeostasis. PAR-2 is activated by serine proteases, such as trypsin, factor Xa, KLKs 4, 5, 6, and 14, and tryptase, as well as membrane-anchored trypsin-like serine proteases, such as matriptase, prostasin, TMPRSS2, hepsin, and human airway trypsin-like protease. ${ }^{17,27,43-46}$ Considering the established role of HAI-1 as a regulator of transmembrane trypsin-like serine protease activities, ${ }^{17,24}$ it is possible to speculate that HAI-1 is a key regulatory molecule that controls PAR-2 activity and, hence, is critical in maintaining epidermal integrity. The current study provided evidence for this hypothesis. However, it remains unclear which protease is directly responsible for the activation of PAR-2 in keratinocytes.

Matriptase is a likely candidate for a PAR-2 activator in the setting of HAI-1 insufficiency because matriptase activity was increased in the supernatant of HAI-1 KD HaCaT cells, and the addition of exogenous matriptase could induce a similar alteration of KIF in control $\mathrm{HaCaT}$ cells. Enhanced pericellular matriptase activity in response to insufficient HAI-1 was also reported in other cell lines. ${ }^{47,48}$ However, matriptase KD only partly alleviated the HAI-1 loss-induced enhancement of p38 phosphorylation. Probably, multiple serine proteases are involved directly or indirectly in the activation of PAR-2 resulting from HAI-1 insufficiency. Prostasin might be an additional candidate, and evidence indicates that there are mutual and complex interactions between matriptase and prostasin for the activation of their zymogens. $^{25,49,50}$

Multiple studies have linked the activation of p38 MAPK to desmosome-associated human skin diseases. For example, pemphigus vulgaris is a skin-blistering disease in humans, and enhanced phosphorylation of p38 MAPK is observed in pemphigus vulgaris skin. ${ }^{51}$ Human keratinocytes treated with pemphigus vulgaris IgG showed p38 activation and retraction of KIF from the cell membrane. ${ }^{5}$ The retraction of KIF was prevented by a p38 MAPK inhibitor. ${ }^{6}$ Similar results were observed in the current study, in which a p38 inhibitor abolished the decreased assembly of KIF to desmosomes induced by HAI-1 loss. In this study, PAR-2 activation induced p38 activation; however, conflicting results have been published regarding PAR-2/p38 signaling, showing both activating and suppressing effects of PAR-2 activation on p38 signaling. For example, trypsin and PAR-2 agonist SLIGKV stimulated a time-dependent increase in p38 MAPK activity in the human keratinocyte cell line NCTC2544, and PAR-2 activation drove pancreatic cancer cell migration via the EGF-Src-Rac-p38 signaling pathway. ${ }^{35,52}$ In contrast, activated protein $\mathrm{C}$-mediated activation of PAR-2 inhibited the phosphorylation of p38 in human primary keratinocytes. ${ }^{53}$ Therefore, p38 signaling, modulated by PAR- 2 activation, may be highly dependent on the cellular context or type.
Desmosomes in keratinocytes are the most important intercellular adherence junctions, because they provide strength to the structure of the epidermis. ${ }^{9}$ Desmogleins are cell adhesion molecules of the desmosome. Previous studies demonstrated that serine proteases are implicated in the cleavage of desmogleins. For example, desmoglein 2 is a functional and physiological substrate of matriptase and KLK- $7,{ }^{10}$ and KLK-5 promotes cleavage of desmoglein- $1 .{ }^{11}$ In the present study, we found that the desmoglein-3 protein level was reduced in HAI-1 KD HaCaT cells without significant alteration of its mRNA level, and treatment with the p38 inhibitor, SB203580, abrogated the reduction of desmoglein-3. However, it remains undetermined whether the decreased desmoglein 3 level simply reflects the decreased number of desmosomes or was caused by enhanced degradation in the absence of HAI-1, leading to a decreased number of desmosomes. Further studies will be needed to evaluate this question.

In summary, our study has demonstrated, for the first time, that HAI-1 regulates KIF assembly into desmosomes through control of PAR-2-dependent p38 MAPK signaling in keratinocytes. This observation provides better understanding of the skin of Spint ${ }^{-1-}$ mice. ${ }^{22}$ Further studies regarding the functions of HAI-1 in human keratinocytes will broaden our understanding of skin disorders associated with disruption of epidermal barrier function.

\section{Acknowledgments}

We thank Dr. Naoki Takeda (Center of Animal Resources and Development, Kumamoto University) for skillful technical assistance to prepare Spint1 ${ }^{-1-}$ mouse epidermis.

M.K. and H.K. conceived and designed the experiments; M.K., A.K., A.S., K.Y., T.F., and H.K. performed the experiments and analyzed data; A.S., T.B., C.-Y.L., and M.D.J. contributed reagents, materials, and analysis tools; and M.K. and H.K. wrote the manuscript.

\section{Supplemental Data}

Supplemental material for this article can be found at http://dx.doi.org/10.1016/j.ajpath.2015.02.009.

\section{References}

1. Madison KC: Barrier function of the skin: "la raison d'être" of the epidermis. J Invest Dermatol 2003, 121:231-241

2. Wan H, Dopping-Hepenstal PJC, Gratian MJ, Stone MG, Zhu G, Purkis PE, South AP, Keane F, Armstrong DKB, Buxton RS, McGrath JA, Eady RAJ: Striate palmoplantar keratoderma arising from desmoplakin and desmoglein 1 mutations is associated with contrasting perturbations of desmosomes and the keratin filament network. Br J Dermatol 2004, 150:878-891

3. Wallace L, Roberts-Thompson L, Reichelt J: Deletion of K1/K10 does not impair epidermal stratification but affects desmosomal structure and nuclear integrity. J Cell Sci 2012, 125:1750-1758 
4. Chamcheu JC, Navsaria H, Pihl-Lundin I, Liovic M, Vahlquist A, Törmä H: Chemical chaperones protect epidermolysis bullosa simplex keratinocytes from heat stress-induced keratin aggregation: involvement of heat shock proteins and MAP kinases. J Invest Dermatol 2011, 131:1684-1691

5. Mao X, Sano Y, Park JM, Payne AS: p38 MAPK activation is downstream of the loss of intercellular adhesion in pemphigus vulgaris. J Biol Chem 2011, 286:1283-1291

6. Berkowitz P, Hu P, Liu Z, Diaz LA, Enghild JJ, Chua MP, Rubenstein DS: Desmosome signaling: inhibition of p38MAPK prevents pemphigus vulgaris IgG-induced cytoskeleton reorganization. J Biol Chem 2005, 280:23778-23784

7. Wang S, Uchi H, Hayashida S, Urabe K, Moroi Y, Furue M: Differential expression of phosphorylated extracellular signal-regulated kinase $1 / 2$, phosphorylated p38 mitogen-activated protein kinase and nuclear factor-kappaB p105/p50 in chronic inflammatory skin diseases. J Dermatol 2009, 36:534-540

8. Wesselborg S: Activation of transcription factor NF-kappa B and p38 mitogen-activated protein kinase is mediated by distinct and separate stress effector pathways. J Biol Chem 1997, 272:12422-12429

9. Kitajima Y: New insights into desmosome regulation and pemphigus blistering as a desmosome-remodeling disease. Kaohsiung J Med Sci 2013, 29:1-13

10. Wadhawan V, Kolhe YA, Sangith N, Gautam AKS, Venkatraman P: From prediction to experimental validation: desmoglein 2 is a functionally relevant substrate of matriptase in epithelial cells and their reciprocal relationship is important for cell adhesion. Biochem $\mathrm{J}$ 2012, 447:61-70

11. Jiang R, Shi Z, Johnson JJ, Liu Y, Stack MS: Kallikrein-5 promotes cleavage of desmoglein-1 and loss of cell-cell cohesion in oral squamous cell carcinoma. J Biol Chem 2011, 286:9127-9135

12. Kataoka H, Suganuma T, Shimomura T, Itoh H, Kitamura N, Nabeshima K, Koono M: Distribution of hepatocyte growth factor activator inhibitor type 1 (HAI-1) in human tissues: cellular surface localization of HAI-1 in simple columnar epithelium and its modulated expression in injured and regenerative tissues. J Histochem Cytochem 1999, 47:673-682

13. Kataoka H, Meng JY, Itoh H, Hamasuna R, Shimomura T, Suganuma T, Koono M: Localization of hepatocyte growth factor activator inhibitor type 1 in Langhans' cells of human placenta. Histochem Cell Biol 2000, 114:469-475

14. Kataoka H, Miyata S, Uchinokura S, Itoh H: Roles of hepatocyte growth factor (HGF) activator and HGF activator inhibitor in the pericellular activation of $\mathrm{HGF} / \mathrm{scatter}$ factor. Cancer Metastasis Rev 2003, 22:223-236

15. Kataoka H, Hamasuna R, Itoh H, Kitamura N, Koono M: Activation of hepatocyte growth factor/scatter factor in colorectal carcinoma. Cancer Res 2000, 60:6148-6159

16. Kataoka H, Kawaguchi M: Hepatocyte growth factor activator (HGFA): pathophysiological functions in vivo. FEBS J 2010, 277: $2230-2237$

17. Antalis TM, Buzza MS, Hodge KM, Hooper JD, Netzel-Arnett S: The cutting edge: membrane-anchored serine protease activities in the pericellular microenvironment. Biochem J 2010, 428:325-346

18. Hashimoto T, Kato M, Shimomura T, Kitamura N: TMPRSS13, a type II transmembrane serine protease, is inhibited by hepatocyte growth factor activator inhibitor type 1 and activates pro-hepatocyte growth factor. FEBS J 2010, 277:4888-4900

19. Kato M, Hashimoto $\mathrm{T}$, Shimomura $\mathrm{T}$, Kataoka $\mathrm{H}$, Ohi $\mathrm{H}$, Kitamura N: Hepatocyte growth factor activator inhibitor type 1 in hibits protease activity and proteolytic activation of human airway trypsin-like protease. J Biochem 2012, 151:179-187

20. Mukai S, Fukushima T, Naka D, Tanaka H, Osada Y, Kataoka H: Activation of hepatocyte growth factor activator zymogen (pro-HGFA) by human kallikrein 1-related peptidases. FEBS J 2008, 275:1003-1017

21. Tanaka H, Nagaike K, Takeda N, Itoh H, Kohama K, Fukushima T, Miyata S, Uchiyama S, Uchinokura S, Shimomura T, Miyazawa K,
Kitamura N, Yamada G, Kataoka H: Hepatocyte growth factor activator inhibitor type 1 (HAI-1) is required for branching morphogenesis in the chorioallantoic placenta. Mol Cell Biol 2005, 25: $5687-5698$

22. Nagaike K, Kawaguchi M, Takeda N, Fukushima T, Sawaguchi A, Kohama K, Setoyama M, Kataoka H: Defect of hepatocyte growth factor activator inhibitor type 1/serine protease inhibitor, Kunitz type 1 (Hai-1/Spint1) leads to ichthyosis-like condition and abnormal hair development in mice. Am J Pathol 2008, 173:1464-1475

23. Kawaguchi M, Takeda N, Hoshiko S, Yorita K, Baba T, Sawaguchi A, Nezu Y, Yoshikawa T, Fukushima T, Kataoka H: Membrane-bound serine protease inhibitor HAI-1 is required for maintenance of intestinal epithelial integrity. Am J Pathol 2011, 179:1815-1826

24. Szabo R, Kosa P, List K, Bugge TH: Loss of matriptase suppression underlies spint1 mutation-associated ichthyosis and postnatal lethality. Am J Pathol 2009, 174:2015-2022

25. Netzel-Arnett S, Currie BM, Szabo R, Lin C-Y, Chen L-M, Chai KX, Antalis TM, Bugge TH, List K: Evidence for a matriptase-prostasin proteolytic cascade regulating terminal epidermal differentiation. J Biol Chem 2006, 281:32941-32945

26. Sales KU, Masedunskas A, Bey AL, Rasmussen AL, Weigert R, List K, Szabo R, Overbeek PA, Bugge TH: Matriptase initiates activation of epidermal pro-kallikrein and disease onset in a mouse model of Netherton syndrome. Nat Genet 2010, 42:676-683

27. Rattenholl A, Steinhoff M: Proteinase-activated receptor-2 in the skin: receptor expression, activation and function during health and disease. Drug News Perspect 2008, 21:369-381

28. Roelandt T, Heughebaert C, Verween G, Giddelo C, Verbeken G, Pirnay J-P, Devos D, Crumrine D, Roseeuw D, Elias PM, Hachem J-P: Actin dynamics regulate immediate PAR-2-dependent responses to acute epidermal permeability barrier abrogation. J Dermatol Sci 2011, 61:101-109

29. Derian CK, Eckardt AJ, Andrade-Gordon P: Differential regulation of human keratinocyte growth and differentiation by a novel family of protease-activated receptors. Cell Growth Differ 1977, 8:743-749

30. Sales KU, Friis S, Konkel JE, Godiksen S, Hatakeyama M, Hansen KK, Rogatto SR, Szabo R, Vogel LK, Chen W, Gutkind JS, Bugge TH: Non-hematopoietic PAR-2 is essential for matriptasedriven pre-malignant progression and potentiation of ras-mediated squamous cell carcinogenesis. Oncogene 2015, 34:346-356

31. Frateschi S, Camerer E, Crisante G, Rieser S, Membrez M, Charles R-P, Beermann F, Stehle J-C, Breiden B, Sandhoff K, Rotman S, Haftek M, Wilson A, Ryser S, Steinhoff M, Coughlin SR, Hummler E: PAR2 absence completely rescues inflammation and ichthyosis caused by altered CAP1/Prss8 expression in mouse skin. Nat Commun 2011, 2:161

32. Chen Y-W, Wang J-K, Chou F-P, Chen C-Y, Rorke EA, Chen LM, Chai KX, Eckert RL, Johnson MD, Lin C-Y: Regulation of the matriptase-prostasin cell surface proteolytic cascade by hepatocyte growth factor activator inhibitor-1 during epidermal differentiation. J Biol Chem 2010, 285:31755-31762

33. McMillan JR, Haftek M, Akiyama M, South AP, Perrot H, McGrath JA, Eady RAJ, Shimizu H: Alterations in desmosome size and number coincide with the loss of keratinocyte cohesion in skin with homozygous and heterozygous defects in the desmosomal protein plakophilin 1. J Invest Dermatol 2003, 121:96-103

34. Kröger C, Loschke F, Schwarz N, Windoffer R, Leube RE, Magin TM: Keratins control intercellular adhesion involving PKC- $\alpha$ mediated desmoplakin phosphorylation. J Cell Biol 2013, 201: 681-692

35. Kanke T, Macfarlane SR, Seatter MJ, Davenport E, Paul A, McKenzie RC, Plevin R: Proteinase-activated receptor-2-mediated activation of stress-activated protein kinases and inhibitory kappa B kinases in NCTC 2544 keratinocytes. J Biol Chem 2001, 276: 31657-31666

36. List K, Haudenschild CC, Szabo R, Chen W, Wahl SM, Swaim W, Engelholm LH, Behrendt N, Bugge TH: Matriptase/MT-SP1 is 
required for postnatal survival, epidermal barrier function, hair follicle development, and thymic homeostasis. Oncogene 2002, 21: 3765-3779

37. Leyvraz C, Charles R-P, Rubera I, Guitard M, Rotman S, Breiden B, Sandhoff K, Hummler E: The epidermal barrier function is dependent on the serine protease CAP1/Prss8. J Cell Biol 2005, 170:487-496

38. Basel-Vanagaite L, Attia R, Ishida-Yamamoto A, Rainshtein L, Ben Amitai D, Lurie R, Pasmanik-Chor M, Indelman M, Zvulunov A, Saban S, Magal N, Sprecher E, Shohat M: Autosomal recessive ichthyosis with hypotrichosis caused by a mutation in ST14, encoding type II transmembrane serine protease matriptase. Am J Hum Genet 2007, 80:467-477

39. List K, Szabo R, Molinolo A, Sriuranpong V, Redeye V, Murdock T, Burke B, Nielsen BS, Gutkind JS, Bugge TH: Deregulated matriptase causes ras-independent multistage carcinogenesis and promotes rasmediated malignant transformation. Genes Dev 2005, 19:1934-1950

40. Hovnanian A: Netherton syndrome: skin inflammation and allergy by loss of protease inhibition. Cell Tissue Res 2013, 351:289-300

41. Carvalho RF, Nilsson G, Harvima IT: Increased mast cell expression of PAR-2 in skin inflammatory diseases and release of IL-8 upon PAR-2 activation. Exp Dermatol 2010, 19:117-122

42. Moniaga CS, Jeong SK, Egawa G, Nakajima S, Hara-Chikuma M, Jeon JE, Lee SH, Hibino T, Miyachi Y, Kabashima K: Protease activity enhances production of thymic stromal lymphopoietin and basophil accumulation in flaky tail mice. Am J Pathol 2013, 182: $841-851$

43. Camerer E, Huang W, Coughlin SR: Tissue factor- and factor Xdependent activation of protease-activated receptor 2 by factor VIIa. Proc Natl Acad Sci U S A 2000, 97:5255-5260

44. Chokki M, Yamamura S, Eguchi H, Masegi T, Horiuchi H, Tanabe H, Kamimura T, Yasuoka S: Human airway trypsin-like protease increases mucin gene expression in airway epithelial cells. Am J Respir Cell Mol Biol 2004, 30:470-478

45. Wilson S, Greer B, Hooper J, Zijlstra A, Walker B, Quigley J, Hawthorne S: The membrane-anchored serine protease, TMPRSS2, activates PAR-2 in prostate cancer cells. Biochem J 2005, 388: 967-972

46. Camerer E, Barker A, Duong DN, Ganesan R, Kataoka H, Cornelissen I, Darragh MR, Hussain A, Zheng Y-W, Srinivasan Y, Brown C, Xu S-M, Regard JB, Lin C-Y, Craik CS, Kirchhofer D, Coughlin SR: Local protease signaling contributes to neural tube closure in the mouse embryo. Dev Cell 2010, 18:25-38

47. Baba T, Kawaguchi M, Fukushima T, Sato Y, Orikawa H, Yorita K, Tanaka H, Lin C-Y, Sakoda S, Kataoka H: Loss of membrane-bound serine protease inhibitor HAI-1 induces oral squamous cell carcinoma cells' invasiveness. J Pathol 2012, 228:181-192

48. Kohama K, Kawaguchi M, Fukushima T, Lin C-Y, Kataoka H: Regulation of pericellular proteolysis by hepatocyte growth factor activator inhibitor type 1 (HAI-1) in trophoblast cells. Hum Cell 2012, 25:100-110

49. Buzza MS, Martin EW, Driesbaugh KH, Désilets A, Leduc R, Antalis TM: Prostasin is required for matriptase activation in intestinal epithelial cells to regulate closure of the paracellular pathway. J Biol Chem 2013, 288:10328-10337

50. Friis S, Sales KU, Schafer JM, Vogel LK, Kataoka H, Bugge TH: The protease inhibitor HAI-2, but not HAI-1, regulates matriptase activation and shedding through prostasin. J Biol Chem 2014, 289: 22319-22332

51. Mavropoulos A, Orfanidou T, Liaskos C, Smyk DS, Spyrou V, Sakkas LI, Rigopoulou EI, Bogdanos DP: p38 MAPK signaling in pemphigus: implications for skin autoimmunity. Autoimmune Dis 2013, 2013:728529

52. Shi K, Queiroz KCS, Stap J, Richel DJ, Spek CA: Protease-activated receptor-2 induces migration of pancreatic cancer cells in an extracellular ATP-dependent manner. J Thromb Haemost 2013, 11: 1892-1902

53. Julovi SM, Xue M, Dervish S, Sambrook PN, March L, Jackson CJ: Protease activated receptor-2 mediates activated protein C-induced cutaneous wound healing via inhibition of p38. Am J Pathol 2011, 179:2233-2242 